\title{
Review Article \\ Toll-Like Receptor 2 as a Regulator of Oral Tolerance in the Gastrointestinal Tract
}

\author{
Matthew C. Tunis ${ }^{1,2}$ and Jean S. Marshall ${ }^{1,2}$ \\ ${ }^{1}$ Department of Microbiology and Immunology, Dalhousie University, 5850 College Street, Halifax, NS, Canada B3H 1 X5 \\ ${ }^{2}$ Dalhousie Inflammation Group, Dalhousie University, 5850 College Street, Halifax, NS, Canada B3H 1 X5
}

Correspondence should be addressed to Jean S. Marshall; jean.marshall@dal.ca

Received 13 June 2014; Revised 2 September 2014; Accepted 4 September 2014; Published 17 September 2014

Academic Editor: Flavio Caprioli

Copyright (C) 2014 M. C. Tunis and J. S. Marshall. This is an open access article distributed under the Creative Commons Attribution License, which permits unrestricted use, distribution, and reproduction in any medium, provided the original work is properly cited.

Food allergy, other adverse immune responses to foods, inflammatory bowel disease, and eosinophilic esophagitis have become increasingly common in the last 30 years. It has been proposed in the "hygiene hypothesis" that dysregulated immune responses to environmental microbial stimuli may modify the balance between tolerance and sensitization in some patients. Of the pattern recognition receptors that respond to microbial signals, toll-like receptors (TLRs) represent the most investigated group. The relationship between allergy and TLR activation is currently at the frontier of immunology research. Although TLR2 is abundant in the mucosal environment, little is known about the complex relationship between bystander TLR2 activation by the commensal microflora and the processing of oral antigens. This review focuses on recent advances in our understanding of the relationship between TLR2 and oral tolerance, with an emphasis on regulatory T cells, eosinophils, B cells, IgA, intestinal regulation, and commensal microbes.

\section{Introduction}

The human intestine is a dynamic environment and host to a myriad of bacteria. It is unclear how these commensals regulate immunologic responses to food antigens, but there is mounting evidence that the microbiological environment of the intestine has a profound influence on oral tolerance [1-5]. In addition to the commensals and pathogens residing in the intestine, food products are often contaminated by a wide array of bacteria and fungi. It is likely that contaminating organisms can shape oral tolerance to foods.

While all microbial pattern recognition receptors (PRRs) are likely to have some relationship to food tolerance and allergen processing, TLR2 may be of unique importance due to its expression by intestinal epithelial cells (IECs) and dendritic cells (DCs) in the intestinal environment. Moreover, a majority of commensal bacteria are Grampositive and thereby have a high capacity for activation of TLR2 $[6,7]$.

TLR2 is important in identifying bacterial [8] and fungal wall components [9], but it must first combine as a heterodimer with TLR1 or TLR6. The TLR1/2 heterodimer responds to triacyl lipopeptides, while the TLR2/6 heterodimer responds to diacyl lipopeptides and peptidoglycan [10]. Both heterodimers of TLR2 signal through the MyD88dependent pathway leading to transcriptional activation of NF- $\kappa \mathrm{B}[11,12]$. TLR2 is expressed by a wide range of cells relevant to mucosal immunity and tolerance including IECs, DCs, T cells, and B cells [13]. While the activation of these TLR2-mediated inflammatory responses is adaptive in the context of pathogenic infection, we are in the early days of understanding how this axis impacts oral tolerance to foods and commensal bacteria. Regulation of IEC permeability $[14,15]$ and the enteric nervous system [16] both rely on TLR2. Furthermore, although the mechanisms are controversial, several studies report exacerbation of inflammatory bowel disease in the absence of TLR2 $[15,17]$. This suggests a critical role for TLR2 in regulating the intestinal microenvironment and local inflammation.

A definitive role for TLR2 expression and activation in the orchestration of tolerance to food antigens has not been characterised. However, growing evidence points to TLR2 as an important factor directing the immunological balance between tolerance and active immune responses to allergens. 


\section{TLR2 Expression and Relationship to Allergy}

TLR2 polymorphisms have been associated with deficits in immune regulation such as inflammatory bowel disease, allergic asthma, and atopic disease [18-21]. Notably, a recent study by Nawijn et al. demonstrated that intranasal TLR2 activation concurrent with aerosolized allergen promoted the expansion of allergen-specific regulatory $\mathrm{T}$ cells (Tregs) and accordingly suppressed asthma in mice [22]. This is consistent with an earlier observation that sublingual TLR2 agonist therapy concurrent with allergen exposure can abrogate airway hyperresponsiveness in mice [23]. A multitude of studies demonstrate that TLR2 stimulation with systemic or mucosal administration of synthetic agonists can prevent antigen presenting cells from eliciting a $\mathrm{T}_{\mathrm{H}} 2$-polarized response, thereby reducing $\operatorname{IgE}$ antibodies and allergenicity in murine asthma models [24-27]. While it is apparent that the delivery of TLR2 agonists via a mucosal route can protect against airway disease in mice, the impacts of TLR2 activation on the processing and tolerance to foods have yet to be characterized. Interestingly, many common foods such as processed meats, chocolate, yoghurt, and cheese contain TLR2 activators [28].

IECs in both the small and large intestine express TLR2, although the distribution and localisation of TLR2 (luminal versus apical) within the cell may vary [29]. In addition, this receptor is expressed by multiple traditional immune effector cells, as described above, often in both intracellular and extracellular compartments. IECs are bathed in an environment replete with TLR2 agonists, such that these cells are probably calibrated to function amid a constitutive level of activation. Accordingly, in TLR2 ${ }^{-/-}$animals, the IEC tight junctions are compromised $[14,15]$. Furthermore, TLR2 stimulation promotes tight junctions [14] that could have implications for food processing and the antigen dosage presented to T cells. Defects in TLR2 are associated with inflammatory bowel disease $[15,17]$ and inappropriate innate responses to intestinal tissue injury in mice [30]. Conversely, intestinal regulation and Treg levels were unchanged in TLR2 ${ }^{-/-}$mice in a chronic model of inflammatory bowel disease [31]. These divergent results may highlight differential roles of TLR2 in chronic inflammation versus acute inflammatory models such as dextran sodium sulphate (DSS) colitis and the different immune cells and mediators driving these models over their respective timeframes $[15,17]$.

It stands to reason that, in the absence of TLR2, the resulting leaky epithelium could allow amplified allergen dosing and exacerbated allergic responses. The impact of this increased permeability during the initial antigen tolerizing stage is not known. Interestingly, it has also been suggested that IECs are largely unresponsive to TLR2 stimulation, despite their TLR2 expression [32]. This is believed to be an adaptive mechanism to prevent uncontrolled intestinal inflammation in response to the constant barrage of TLR2 agonists from Gram-positive commensals. Consistent with this, TLR2 expression is higher in IECs of the colon compared to the small intestine where earlier food antigen exposure occurs and oral tolerance is thought to be established.
TLR2 functions both as a heterodimer with TLR1 or TLR6 and also potentially as a homodimer. $\mathrm{Pam}_{3} \mathrm{CSK}_{4}$ is a selective ligand for the TLR1/TLR2 heterodimer, while TLR6/TLR1 responds to other ligands such as FSL-1. The distinct role of TLR6 is of particular interest since it is expressed on only selected cell types in the intestine, while TLR1 is much more widely expressed. In a very recent study [33], cells from the intestinal lymphoid tissues activated with anti-CD3 were shown to be more effectively polarized towards $\mathrm{T}_{\mathrm{H}} 17$ and $\mathrm{T}_{\mathrm{H}} 1$ responses by treatment with FSL-1 than with $\mathrm{Pam}_{3} \mathrm{CSK}_{4}$. Using a DSS model of colitis, the TLR6 deficient animals were shown to be disease resistant. In parallel studies of human tissues, TLR6 expression was found to be correlated with the levels of RORC mRNA in inflamed intestines of IBD patients. These results could suggest a role for TLR6 in IBD therapy and have potential implications for the development of $\mathrm{T}$ cell responses in the context of TLR6 activators. Clearly, the roles of TLR signaling in the context of inflammatory intestinal disease are not limited to the TLR2 molecule alone.

\section{TLR2 and Eosinophil Responses}

Eosinophils represent an important aspect of chronic allergic disease, and TLR2 may have a key relationship to eosinophils in the mucosal environment within the context of allergy and gastrointestinal inflammation. In animal studies, TLR2 expression and activation were sufficient to facilitate eosinophil recruitment and tissue eosinophilia of the large intestine in the context of experimental colitis [34]. Similarly, eosinophil recruitment to the large intestine and the subsequent chronic inflammatory responses were TLR2dependent during parasitic Schistosoma mansoni infection in mice [35].

A causal link has not been established, but patients with eosinophilic gastrointestinal diseases experience elevated rates of asthma and allergy with up to $76 \%$ of patients testing positive for food allergen skin pricks [36]. IgE class switch recombination and local IgE production are also both significantly higher in patients with eosinophilic esophagitis [37]. By contrast, the mucosal administration of a synthetic TLR2 agonist in the airways reliably reduced eosinophilia of the lungs in murine asthma models [24, 26, 38]. TLR2 stimulation therefore has different outcomes on eosinophil tissue homing depending on the activation site and inflammatory status of the tissue in question. The induction of TLR2dependent eosinophil homing to the intestine may impact the $\mathrm{T}_{\mathrm{H}} 2$ polarization of antigen responses and ultimately alter allergic inflammation or the ongoing regulation of responses to food and the microflora within this compartment.

\section{TLR2 and the Enteric Nervous System}

The interplay between the nervous system and the immune system can be critical for homeostasis and effective immunity. This is particularly true in the intestine where the enteric nervous system (ENS) modifies intestinal motility and epithelial barrier function. TLR2 has been shown to be expressed on enteric neurons, glia, and smooth muscle cells of the intestinal wall. TLR2 $2^{-/-}$mice demonstrated disrupted ENS architecture 
as well as intestinal dysmotility that could be corrected by the addition of glial cell line-derived neurotrophic factor (GDNF). The increased susceptibility to DSS colitis exhibited by TLR $2^{-/-}$mice can be abrogated by treatment with GDNF. Notably, wild type mice depleted of intestinal microbiota had similar defects in the ENS and intestinal motility to mice deficient in TLR2 [16]. It is not yet clear whether the substantial impact of TLR2 deficiency on ENS function is direct or via secondary effects on the microbiota. However, TLR2 has been implicated in the response to nerve injury in other tissues via the action of local macrophages [39], confirming the importance of this receptor to neuronal function regardless of microbial influences.

\section{A Relationship between TLR2, Tregs, Microbes, and Tolerance}

Oral tolerance can be defined as antigen-specific humoral and cellular hyporesponsiveness following oral antigen exposure $[40,41]$. Tolerance is readily induced in mice and humans following oral treatment with food antigen, and food allergy is often considered to result from a failure of oral tolerance mechanisms. The complex process of oral tolerance is known to involve several different cell subsets within the gut associated lymphoid tissues [42], perhaps most notably the Tregs which are required for the induction and maintenance of tolerance to foods [43-46]. Tregs are therefore positioned to profoundly alter the nature of responses to food antigen. Several studies have directly investigated the impact of TLR2 activation on $\mathrm{T}$ cell homing and function. Wang et al. demonstrated that TLR2 and MyD88 are necessary for DCs to imprint T cells with intestinal homing markers $\alpha_{4} \beta_{7}$ and CCR9 [47]. This homing is pertinent in light of evidence that Treg intestinal homing to the lamina propria is essential for the ongoing maintenance of oral tolerance to foods [44]. Importantly, lamina propria DC subsets were shown to have high expression of TLR2 relative to other lymphatic DC populations [48]. However, among lamina propria DCs, the $\mathrm{CD}_{103}{ }^{+}$cells, known to be tolerogenic, had lower TLR2 expression compared to other subpopulations. This suggests that TLR2 expression on DCs may not be necessary to drive Treg differentiation.

The expression of Foxp3, associated with Treg development, is abrogated by TLR2 signaling events within the T cell [49]. Similarly, TLR2 activation with the lipopeptide $\mathrm{Pam}_{3} \mathrm{CSK}_{4}$ can abrogate the suppressive capacity of Tregs and DCs in vitro [50-52]. Paradoxically, a systemic administration of $\mathrm{Pam}_{3} \mathrm{CSK}_{4}$ promoted the expansion of adoptively transferred Tregs in vivo but mitigated their suppressive activity in mice [50]. It may be that the source of Tregs, natural or inducible, impacts the nature and sensitivity of responses to TLR2 stimulation.

It is difficult to reconcile the data above regarding TLR2 abrogation of Treg function, which may be most relevant to oral tolerance induction, with the observation that TLR2 can support the induction of Tregs in the context of commensal microbes; but there is mounting evidence that TLR2 activation by intestinal commensal bacteria can promote local regulatory responses. Microbiota are important for the appropriate maturation of intestinal immunity and this can complicate the interpretation of experimental studies examining the role of bacterial flora in specific immune responses. However, elegant studies with Bacteroides fragilis in mice have shown that Tregs induced by TLR 2 activation with the unique bacterial polysaccharide A are necessary for successful intestinal colonization [53, 54]. Similarly, the probiotic Bifidobacterium infantis promotes Tregs and regulatory cytokine production in humans and functions via TLR2 [55]. It was also recently demonstrated that probiotic Bifidobacterium breve induces regulatory IL-10 secreting Tr1 cells via TLR2 stimulation of $\mathrm{CD} 03^{+}$dendritic cells, thereby reducing inflammation in the large intestine [56]. Treatment with Bifidobacterium components or the TLR2 activation of mast cells by $\mathrm{Pam}_{3} \mathrm{CSK}_{4}$ has even been reported to suppress IgE-mediated mast cell degranulation in vitro and in vivo [57].

While recent studies show a clear relationship between some commensals and immunologic tolerance, the antigenspecificity of these Treg responses has not been adequately characterized. Moreover, studies exploring commensal Treg induction and the resulting suppression of inflammation tend to examine responses in the colon and cecum, while little attention has been paid to the relationship between commensals and Tregs in the small intestine. The small intestine is an important site of food tolerance induction, and few studies have addressed the role of commensal colonization on food allergy. We do know that commensal bacteria are required for appropriate levels of Tregs to be established in the MLN, and without them oral tolerance is inadequate as shown by studies in germ-free (GF) mice [58]. Furthermore, it has been proposed that the inability of GF mice to establish oral tolerance may be directly related to the failure of these mice to establish adequate $\mathrm{T}$ cell populations in the PPs [59]. Several studies have also shown that GF mice display a more $\mathrm{T}_{\mathrm{H}} 2$ polarized response to oral antigens, resulting in IgE antibody production specific to oral antigen and a failure to be tolerized [60-62]. A recent study by Noval Rivas et al. demonstrated that variations in the murine commensal flora will dictate the balance between oral tolerance and allergy to oral antigen through involvement of Treg populations [5]. The changes in Treg and humoral responses to food antigen in the context of commensals are likely to implicate TLRs, but more directed investigations must be carried out to fully understand the precise role of TLR2 signaling in food tolerance.

\section{TLR2 Directs B Cell and IgA Responses}

IgA is the most abundant mucosal antibody, with an average of $5 \mathrm{~g}$ secreted daily in human feces [63]. IgA occurs both as a monomer in serum and as a dimer bound by the Jchain. The IgA dimers are translocated to the gut lumen and to other mucosal surfaces by the poly-Ig receptor (pIgR) on IECs, where the antibodies participate in the immune exclusion of microbes. The relationship between secreted IgA and food allergy has not been fully elucidated, but patients with selective IgA deficiency demonstrate impaired mucosal immunity and deficits of intestinal regulation that correlate with higher rates of food allergy and inflammatory bowel 


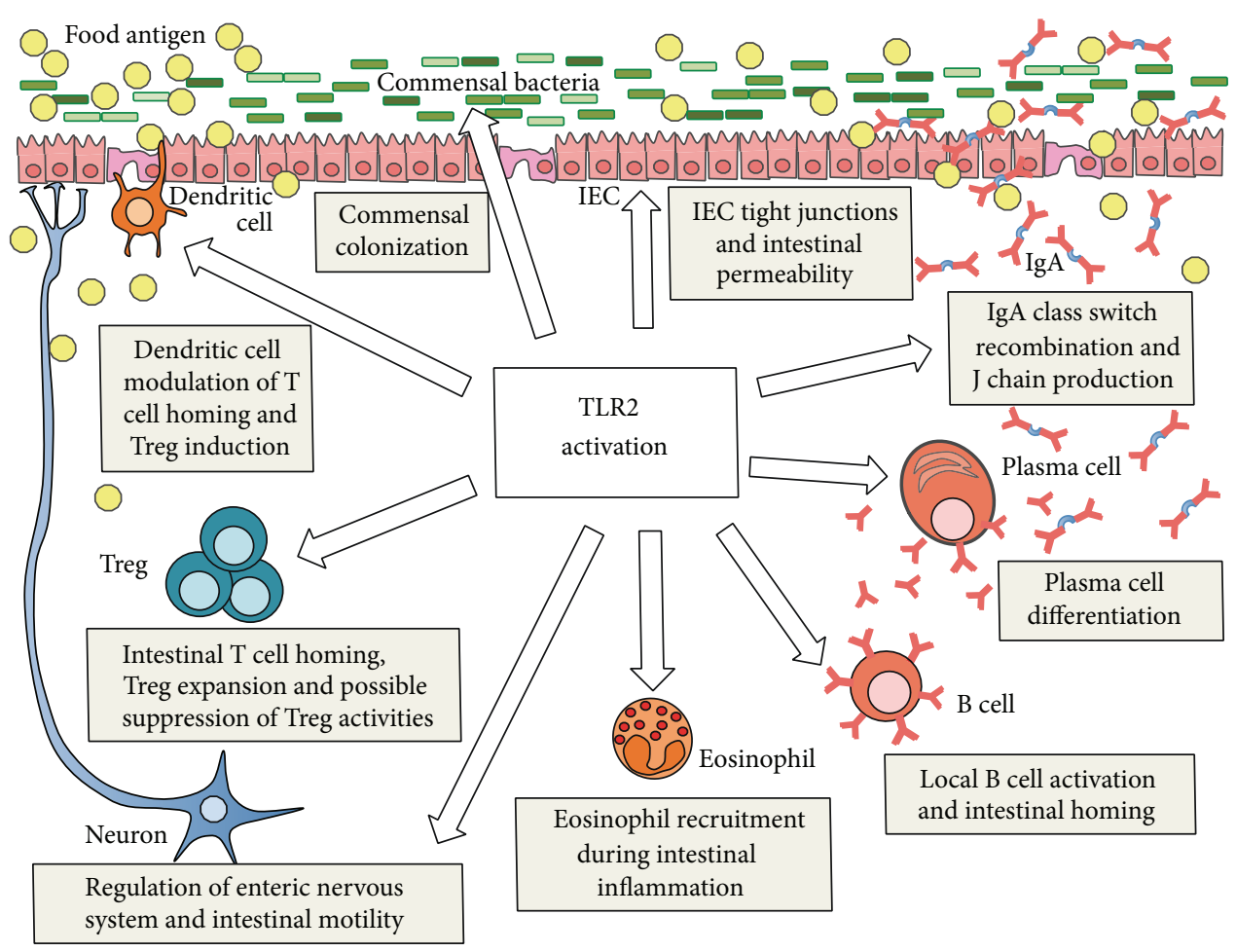

FIGURE 1: Proposed involvement of TLR2 in oral antigen responses of the intestinal microenvironment. This figure outlines several suggested roles of key intestinal cell types in the regulation of oral tolerance to oral antigens in the context of TLR2 activation.

disease $[64,65]$. Further to this, secreted IgA has been correlated with improved tolerance to peanut challenge in allergic patients [66]. Both serum and secreted antigen-specific IgA have been shown to prevent oral anaphylaxis [67] and allergic diarrhea [68] in mice, suggesting that $\operatorname{IgA}$ responses can be protective in the context of an oral allergen challenge. Elevated secreted IgA has been documented in mice treated with oral food antigen compared to naïve animals [69], and antigen-specific IgA is detected in the serum of mice upon oral immunotherapy treatment [70]. Thus, it appears likely that robust $\operatorname{IgA}$ production is related to protection against allergic responses to food. TLR2 stimulation has well-documented effects on B cell activation and local IgA responses.

Both naïve and activated B cells express TLR2 [71]. Therefore, in addition to activating IECs, DCs, and T cells in the mucosal environment, TLR2 ligands can act directly on B cells. It was recently reported that TLR2 activation of resting murine $B$ cells in concert with CD40L stimulation can dramatically enhance proliferation, class switch recombination, and plasma cell differentiation [72, 73]. Work by Jain et al. has also shown that TLR2 activation of B cells enhanced their ability to respond to CD40 stimulation by $\mathrm{T}$ cells upon antigen presentation [73]. Consistent with this, $\mathrm{Pam}_{3} \mathrm{CSK}_{4}$ treatment of naïve human peripheral B cells results in production of IL-6 and IL-13 [74], both of which can promote $\mathrm{B}$ cell activation and antibody production. Of particular relevance to oral tolerance, TLR2 stimulation of B cells with synthetic lipopeptide resulted in the proliferation of Peyer's patch B cells and subsequent antibody production in a murine model [71]. Furthermore, stimulation of human B cells with TLR2 agonists promotes IgA production, J chain production, and the expression of intestinal homing markers CCR9 and CCR10 [75]. Prior to the characterization of TLR2, an older study with lipopeptides found that oral administration of $\mathrm{Pam}_{3} \mathrm{CSK}_{4}$ (now known to be a TLR2/1 agonist) concurrent with oral antigen promoted significant antigen-specific plasma IgA and secretory fecal IgA responses in a murine model [76]. Finally, expression of the $\mathrm{pIgR}$ and transcytosis of IgA dimers across IECs are impaired in the absence of MyD88 signaling [77]. Taken together, these findings identify key roles for TLR2 in regulating B cell maturation, expansion, homing, IgA production, and even IgA secretion.

Evidence points towards B cell activation and IgA production as necessary to contain commensal microbes to the intestinal lumen $[78,79]$. The same principle may apply to food antigens, but the antigen-specificity of activated $\mathrm{B}$ cells is at issue. Further investigation is necessary to elucidate whether bystander TLR2 activation of intestinal B cells by commensal bacteria or food contaminants is capable of promoting expansion of food-specific $B$ cells and the associated $\operatorname{IgA}$ response.

\section{Summary}

TLR2 is increasingly at the forefront of intestinal immunology investigations. TLR2 stimulation promotes intestinal barrier function, B cell maturation, mucosal homing, and IgA responses (Figure 1). TLR2 activation by some commensal 
species facilitates Treg differentiation. However, most reports indicate that the direct impact of TLR2 stimulation on Tregs is to suppress their function once induced, and systemic TLR2 activation promotes intestinal homing of eosinophils during intestinal inflammation and impacts enteric nerve function (Figure 1). It is likely that the TLR2-dependent axis of regulation and allergic sensitization is plastic and responsive to changes in TLR2 agonist dosing. Furthermore, the physiological site of activation may be critically important in dictating subsequent responses. For example, constitutive low grade commensal TLR2 stimulation may support tolerance to foods, but a breach in the mucosal barrier and amplified TLR2 agonist dosing may promote local inflammation and sensitization to bystander food antigens. Such a scenario needs to be tested experimentally in order to better understand the relationship between TLR2 and food allergy. Finally, a dedicated comparison between TLR2 activation in the small and large intestine and the subsequent Treg and B cell responses would be extremely important for understanding the implications of TLR2 in food tolerance and allergy. There is a true deficit of small intestine research in this field.

Unfortunately, the most fundamental question remains unanswered: does TLR2 activation support or disrupt human oral tolerance to food antigens? As described above, a number of lines of evidence suggest that this may be the case, but there is insufficient evidence available to move forward with TLR2 targeted prevention or treatment strategies. With the current interest in host-commensal interactions and the growing importance of food allergy, we are sure to see rapid advancements in this area that will have implications both for allergic disease and for effective oral immunization.

\section{Conflict of Interests}

The authors have no conflict of interests.

\section{Acknowledgments}

This work was supported by AllerGen NCE Inc.

\section{References}

[1] P. M. Matricardi, F. Rosmini, S. Riondino et al., "Exposure to foodborne and orofecal microbes versus airborne viruses in relation to atopy and allergic asthma: epidemiological study," British Medical Journal, vol. 320, no. 7232, pp. 412-417, 2000.

[2] R. M. Helm and A. W. Burks, "Mechanisms of food allergy," Current Opinion in Immunology, vol. 12, no. 6, pp. 647-653, 2000.

[3] S. Romagnani, "The increased prevalence of allergy and the hygiene hypothesis: missing immune deviation, reduced immune suppression, or both?" Immunology, vol. 112, no. 3, pp. 352-363, 2004.

[4] S. F. Bloomfield, R. Stanwell-Smith, R. W. R. Crevel, and J. Pickup, "Too clean, or not too clean: the hygiene hypothesis and home hygiene," Clinical and Experimental Allergy, vol. 36, no. 4, pp. 402-425, 2006.

[5] M. Noval Rivas, O. T. Burton, P. Wise et al., "A microbiota signature associated with experimental food allergy promotes allergic sensitization and anaphylaxis," Journal of Allergy and Clinical Immunology, vol. 131, no. 1, pp. 201-212, 2013.

[6] P. B. Eckburg, E. M. Bik, C. N. Bernstein et al., "Diversity of the human intestinal microbial flora," Science, vol. 308, no. 5728, pp. 1635-1638, 2005.

[7] J. Qin, R. Li, J. Raes et al., "A human gut microbial gene catalogue established by metagenomic sequencing," Nature, vol. 464, no. 7285, pp. 59-65, 2010.

[8] O. Takeuchi, A. Kaufmann, K. Grote et al., "Cutting edge: preferentially the $R$-stereoisomer of the mycoplasmal lipopeptide macrophage-activating lipopeptide- 2 activates immune cells through a toll-like receptor 2- and MyD88-dependent signaling pathway," The Journal of Immunology, vol. 164, no. 2, pp. 554557, 2000.

[9] D. M. Underhill, A. Ozinsky, A. M. Hajjar et al., "The tolllike receptor 2 is recruited to macrophage phagosomes and discriminates between pathogens," Nature, vol. 401, no. 6755, pp. 811-815, 1999.

[10] M. S. Jin and J. O. Lee, "Structures of the toll-like receptor family and its ligand complexes," Immunity, vol. 29, no. 2, pp. 182-191, 2008.

[11] T. Kawai and S. Akira, “TLR signaling," Cell Death and Differentiation, vol. 13, no. 5, pp. 816-825, 2006.

[12] T. Kawai and S. Akira, "The role of pattern-recognition receptors in innate immunity: update on toll-like receptors," Nature Immunology, vol. 11, no. 5, pp. 373-384, 2010.

[13] H. H. Oberg, M. Juricke, D. Kabelitz, and D. Wesch, "Regulation of T cell activation by TLR ligands," European Journal of Cell Biology, vol. 90, no. 6-7, pp. 582-592, 2011.

[14] E. Cario, G. Gerken, and D. K. Podolsky, “Toll-like receptor 2 controls mucosal inflammation by regulating epithelial barrier function," Gastroenterology, vol. 132, no. 4, pp. 1359-1374, 2007.

[15] E. Cario, "Barrier-protective function of intestinal epithelial Toll-like receptor 2," Mucosal Immunology, vol. 1, no. 1, pp. S62S66, 2008.

[16] P. Brun, M. C. Giron, M. Qesari et al., “Toll-like receptor 2 regulates intestinal inflammation by controlling integrity of the enteric nervous system," Gastroenterology, vol. 145, no. 6, pp. 1323-1333, 2013.

[17] E. J. Albert and J. S. Marshall, "Aging in the absence of TLR2 is associated with reduced IFN- $\gamma$ responses in the large intestine and increased severity of induced colitis," Journal of Leukocyte Biology, vol. 83, no. 4, pp. 833-842, 2008.

[18] M. Pierik, S. Joossens, K. van Steen et al., “Toll-like receptor$1,-2$, and -6 polymorphisms influence disease extension in inflammatory bowel diseases," Inflammatory Bowel Diseases, vol. 12, no. 1, pp. 1-8, 2006.

[19] D.-Y. Oh, R. R. Schumann, L. Hamann, K. Neumann, M. Worm, and G. Heine, "Association of the toll-like receptor 2 A-16934T promoter polymorphism with severe atopic dermatitis," Allergy, vol. 64, no. 11, pp. 1608-1615, 2009.

[20] W. Eder, W. Klimecki, L. Yu et al., "Toll-like receptor 2 as a major gene for asthma in children of European farmers," Journal of Allergy and Clinical Immunology, vol. 113, no. 3, pp. 482-488, 2004.

[21] M. Bjørnvold, M. C. Munthe-Kaas, T. Egeland et al., "A TLR2 polymorphism is associated with type 1 diabetes and allergic asthma," Genes and Immunity, vol. 10, no. 2, pp. 181-187, 2009.

[22] M. C. Nawijn, A. C. Motta, R. Gras, S. Shirinbak, H. Maazi, and A. J. M. van Oosterhout, "TLR-2 activation induces regulatory $\mathrm{T}$ cells and long-term suppression of asthma manifestations in mice," PLoS ONE, vol. 8, no. 2, Article ID e55307, 2013. 
[23] V. Lombardi, L. van Overtvelt, S. Horiot et al., “Toll-like receptor 2 agonist Pam3CSK4 enhances the induction of antigen-specific tolerance via the sublingual route," Clinical and Experimental Allergy, vol. 38, no. 11, pp. 1819-1829, 2008.

[24] C. Zhou, X.-D. Kang, and Z. Chen, "A synthetic toll-like receptor 2 ligand decreases allergic immune responses in a mouse rhinitis model sensitized to mite allergen," Journal of Zhejiang University: Science B, vol. 9, no. 4, pp. 279-285, 2008.

[25] M. Patel, D. Xu, P. Kewin et al., “TLR2 agonist ameliorates established allergic airway inflammation by promoting Thl response and not via regulatory T cells," Journal of Immunology, vol. 174, no. 12, pp. 7558-7563, 2005.

[26] B. Fuchs, S. Knothe, S. Rochlitzer et al., "A toll-like receptor $2 / 6$ agonist reduces allergic airway inflammation in chronic respiratory sensitisation to timothy grass pollen antigens," International Archives of Allergy and Immunology, vol. 152, no. 2, pp. 131-139, 2010.

[27] R. C. Taylor, P. Richmond, and J. W. Upham, "Toll-like receptor 2 ligands inhibit TH2 responses to mite allergen," Journal of Allergy and Clinical Immunology, vol. 117, no. 5, pp. 1148-1154, 2006.

[28] C. Erridge, "The capacity of foodstuffs to induce innate immune activation of human monocytes in vitro is dependent on food content of stimulants of Toll-like receptors 2 and 4," British Journal of Nutrition, vol. 105, no. 1, pp. 15-23, 2011.

[29] M. T. Abreu, M. Fukata, and M. Arditi, “TLR signaling in the gut in health and disease," Journal of Immunology, vol. 174, no. 8, pp. 4453-4460, 2005.

[30] C. J. Aprahamian, R. G. Lorenz, C. M. Harmon, and R. A. Dimmit, "Toll-like receptor 2 is protective of ischemiareperfusion-mediated small-bowel injury in a murine model," Pediatric Critical Care Medicine, vol. 9, no. 1, pp. 105-109, 2008.

[31] O. Boulard, M. J. Asquith, F. Powrie, and K. J. Maloy, "TLR2independent induction and regulation of chronic intestinal inflammation," European Journal of Immunology, vol. 40, no. 2, pp. 516-524, 2010.

[32] G. Melmed, L. S. Thomas, N. Lee et al., "Human intestinal epithelial cells are broadly unresponsive to toll-like receptor 2-dependent bacterial ligands: implications for host-microbial interactions in the gut," Journal of Immunology, vol. 170, no. 3, pp. 1406-1415, 2003.

[33] M. E. Morgan, P. J. Koelink, B. Zheng et al., "Toll-like receptor 6 stimulation promotes T-helper 1 and 17 responses in gastrointestinal-associated lymphoid tissue and modulates murine experimental colitis," Mucosal Immunology, vol. 7, no. 5, pp. 1266-1277, 2014.

[34] E. J. Albert, J. Duplisea, W. Dawicki, I. D. Haidl, and J. S. Marshall, "Tissue eosinophilia in a mouse model of colitis is highly dependent on TLR2 and independent of mast cells," The American Journal of Pathology, vol. 178, no. 1, pp. 150-160, 2011.

[35] K. G. Magalhães, P. E. Almeida, G. C. Atella et al., "Schistosomal-derived lysophosphatidylcholine are involved in eosinophil activation and recruitment through toll-like receptor-2-dependent mechanisms," Journal of Infectious Diseases, vol. 202, no. 9, pp. 1369-1379, 2010.

[36] H. Appelman, "Clinical, pathologic, and molecular characterization of familial eosinophilic esophagitis compared with sporadic cases," Clinical Gastroenterology and Hepatology, vol. 6, no. 11, p. 1283, 2008.

[37] M. Vicario, C. Blanchard, K. F. Stringer et al., "Local B cells and IgE production in the oesophageal mucosa in eosinophilic oesophagitis," Gut, vol. 59, no. 1, pp. 12-20, 2010.
[38] J. K. Krishnaswamy, A. C. Jirmo, A. M. Baru et al., “Toll-like receptor-2 agonist-allergen coupling efficiently redirects Th2 cell responses and inhibits allergic airway eosinophilia," The American Journal of Respiratory Cell and Molecular Biology, vol. 47, no. 6, pp. 852-863, 2012.

[39] X. Q. Shi, H. Zekki, and J. Zhang, "The role of TLR2 in nerve injury-induced neuropathic pain is essentially mediated through macrophages in peripheral inflammatory response," Glia, vol. 59, no. 2, pp. 231-241, 2011.

[40] A. M. C. Faria and H. L. Weiner, "Oral tolerance," Immunological Reviews, vol. 206, pp. 232-259, 2005.

[41] B. P. Vickery and A. W. Burks, "Immunotherapy in the treatment of food allergy: focus on oral tolerance," Current Opinion in Allergy and Clinical Immunology, vol. 9, no. 4, pp. 364-370, 2009.

[42] A. M. Mowat, "Anatomical basis of tolerance and immunity to intestinal antigens," Nature Reviews Immunology, vol. 3, no. 4, pp. 331-341, 2003.

[43] H. L. Weiner, A. P. da Cunha, F. Quintana, and H. Wu, "Oral tolerance," Immunological Reviews, vol. 241, no. 1, pp. 241-259, 2011.

[44] U. Hadis, B. Wahl, O. Schulz et al., "Intestinal tolerance requires gut homing and expansion of FoxP3+ regulatory $\mathrm{T}$ cells in the lamina propria," Immunity, vol. 34, no. 2, pp. 237-246, 2011.

[45] A. Krogulska, M. Borowiec, E. Polakowska, J. Dynowski, W. Młynarski, and K. Wasowska-Królikowska, "FOXP3, IL-10, and TGF- $\beta$ genes expression in children with IgE-dependent food allergy," Journal of Clinical Immunology, vol. 31, no. 2, pp. 205215, 2011.

[46] H. Yamashita, K. Takahashi, H. Tanaka, H. Nagai, and N. Inagaki, "Overcoming food allergy through acquired tolerance conferred by transfer of Tregs in a murine model," Allergy, vol. 67, no. 2, pp. 201-209, 2012.

[47] S. Wang, E. J. Villablanca, J. De Calisto et al., "MyD88-dependent TLR1/2 signals educate dendritic cells with gut-specific imprinting properties," Journal of Immunology, vol. 187, no. 1, pp. 141-150, 2011.

[48] I. Monteleone, A. M. Platt, E. Jaensson, W. W. Agace, and A. M. Mowat, "IL-10-dependent partial refractoriness to Tolllike receptor stimulation modulates gut mucosal dendritic cell function," European Journal of Immunology, vol. 38, no. 6, pp. 1533-1547, 2008.

[49] G. Lal, N. Yin, J. Xu et al., "Distinct inflammatory signals have physiologically divergent effects on epigenetic regulation of Foxp3 expression and Treg function," The American Journal of Transplantation, vol. 11, no. 2, pp. 203-214, 2011.

[50] R. P. M. Sutmuller, M. H. M. G. M. Den Brok, M. Kramer et al., "Toll-like receptor 2 controls expansion and function of regulatory T cells," Journal of Clinical Investigation, vol. 116, no. 2, pp. 485-494, 2006.

[51] W. W. C. van Maren, S. Nierkens, L. W. Toonen, J. M. Bolscher, R. P. M. Sutmuller, and G. J. Adema, "Multifaceted effects of synthetic TLR2 ligand and Legionella pneumophilia on Tregmediated suppression of T cell activation," BMC Immunology, vol. 12, article 23, 2011.

[52] H. Liu, M. Komai-Koma, D. Xu, and F. Y. Liew, "Toll-like receptor 2 signaling modulates the functions of CD4+ CD25+ regulatory T cells," Proceedings of the National Academy of Sciences of the United States of America, vol. 103, no. 18, pp. 7048-7053, 2006.

[53] J. L. Round and S. K. Mazmanian, "Inducible Foxp3+ regulatory T-cell development by a commensal bacterium of the intestinal 
microbiota," Proceedings of the National Academy of Sciences of the United States of America, vol. 107, no. 27, pp. 12204-12209, 2010.

[54] J. L. Round, S. M. Lee, J. Li et al., "The toll-like receptor 2 pathway establishes colonization by a commensal of the human microbiota," Science, vol. 332, no. 6032, pp. 974-977, 2011.

[55] P. Konieczna, D. Groeger, M. Ziegler et al., "Bifidobacterium infantis 35624 administration induces Foxp3 T regulatory cells in human peripheral blood: potential role for myeloid and plasmacytoid dendritic cells," Gut, vol. 61, no. 3, pp. 354-366, 2012.

[56] S. G. Jeon, H. Kayama, Y. Ueda et al., "Probiotic Bifidobacterium breve induces IL-10-producing Tr1 cells in the colon," PLoS Pathogens, vol. 8, no. 5, Article ID e1002714, 2012.

[57] K. Kasakura, K. Takahashi, T. Aizawa, A. Hosono, and S. Kaminogawa, "A TLR2 ligand suppresses allergic inflammatory reactions by acting directly on mast cells," International Archives of Allergy and Immunology, vol. 150, no. 4, pp. 359-369, 2009.

[58] H. Ishikawa, K. Tanaka, Y. Maeda et al., "Effect of intestinal microbiota on the induction of regulatory CD25+CD4+ T cells," Clinical and Experimental Immunology, vol. 153, no. 1, pp. 127-135, 2008.

[59] Y. Maeda, S. Noda, K. Tanaka et al., "The failure of oral tolerance induction is functionally coupled to the absence of T cells in Peyer's patches under germfree conditions," Immunobiology, vol. 204, no. 4, pp. 442-457, 2001.

[60] N. Sudo, S. A. Sawamura, K. Tanaka, Y. Aiba, C. Kubo, and Y. Koga, "The requirement of intestinal bacterial flora for the development of an IgE production system fully susceptible to oral tolerance induction," Journal of Immunology, vol. 159, no. 4, pp. 1739-1745, 1997.

[61] K. Tanaka and H. Ishikawa, "Role of intestinal bacterial flora in oral tolerance induction," Histology and Histopathology, vol. 19, no. 3, pp. 907-914, 2004.

[62] J. Cahenzli, Y. Köller, M. Wyss, M. B. Geuking, and K. D. McCoy, "Intestinal microbial diversity during early-life colonization shapes long-term IgE levels," Cell Host and Microbe, vol. 14, no. 5, pp. 559-570, 2013.

[63] W. Su, J. N. Gordon, F. Barone et al., "Lambda light chain revision in the human intestinal $\operatorname{IgA}$ response," Journal of Immunology, vol. 181, no. 2, pp. 1264-1271, 2008.

[64] A. H. A. Latiff and M. A. Kerr, "The clinical significance of immunoglobulin A deficiency," Annals of Clinical Biochemistry, vol. 44, part 2, pp. 131-139, 2007.

[65] M. Janzi, I. Kull, R. Sjöberg et al., "Selective IgA deficiency in early life: association to infections and allergic diseases during childhood," Clinical Immunology, vol. 133, no. 1, pp. 78-85, 2009.

[66] M. Kulis, K. Saba, E. H. Kim et al., "Increased peanut-specific IgA levels in saliva correlate with food challenge outcomes after peanut sublingual immunotherapy," Journal of Allergy and Clinical Immunology, vol. 129, no. 4, pp. 1159-1162, 2012.

[67] R. T. Strait, A. Mahler, S. Hogan, M. Khodoun, A. Shibuya, and F. D. Finkelman, "Ingested allergens must be absorbed systemically to induce systemic anaphylaxis," Journal of Allergy and Clinical Immunology, vol. 127, no. 4, pp. 982.e1-989.e1, 2011.

[68] Z. Y. Kucuk, R. Strait, M. V. Khodoun, A. Mahler, S. Hogan, and F. D. Finkelman, "Induction and suppression of allergic diarrhea and systemic anaphylaxis in a murine model of food allergy," Journal of Allergy and Clinical Immunology, vol. 129, no. 5, pp. 1343-1348, 2012.
[69] C. P. Frossard, C. Hauser, and P. A. Eigenmann, "Antigenspecific secretory IgA antibodies in the gut are decreased in a mouse model of food allergy," Journal of Allergy and Clinical Immunology, vol. 114, no. 2, pp. 377-382, 2004.

[70] S. A. Leonard, G. Martos, W. Wang, A. Nowak-Wegrzyn, and M. C. Berin, "Oral immunotherapy induces local protective mechanisms in the gastrointestinal mucosa," Journal of Allergy and Clinical Immunology, vol. 129, no. 6, pp. 1579-1587, 2012.

[71] M. Gururajan, J. Jacob, and B. Pulendran, “Toll-like receptor expression and responsiveness of distinct murine splenic and mucosal B-cell subsets," PLoS ONE, vol. 2, no. 9, article e863, 2007.

[72] E. Boeglin, C. R. Smulski, S. Brun, S. Milosevic, P. Schneider, and S. Fournel, "Toll-Like receptor agonists synergize with CD40L to induce either proliferation or plasma cell differentiation of mouse B cells," PLoS ONE, vol. 6, no. 10, Article ID e25542, 2011.

[73] S. Jain, S. B. Chodisetti, and J. N. Agrewala, "CD40 signaling synergizes with TLR-2 in the BCR independent activation of resting B cells," PLoS ONE, vol. 6, no. 6, Article ID e20651, 2011.

[74] S. Agrawal and S. Gupta, "TLR1/2, TLR7, and TLR9 signals directly activate human peripheral blood naive and memory $\mathrm{B}$ cell subsets to produce cytokines, chemokines, and hematopoietic growth factors," Journal of Clinical Immunology, vol. 31, no. 1, pp. 89-98, 2011.

[75] Y. Liang, H. Hasturk, J. Elliot et al., “Toll-like receptor 2 induces mucosal homing receptor expression and IgA production by human B cells," Clinical Immunology, vol. 138, no. 1, pp. 33-40, 2011.

[76] M. Huber, W. Baier, W. G. Bessler, and L. Heinevetter, "Modulation of the Th1/Th2 bias by lipopeptide and saponin adjuvants in orally immunized mice," Immunobiology, vol. 205, no. 1, pp. 61-73, 2002.

[77] A. L. Frantz, E. W. Rogier, C. R. Weber et al., "Targeted deletion of MyD88 in intestinal epithelial cells results in compromised antibacterial immunity associated with downregulation of polymeric immunoglobulin receptor, mucin-2, and antibacterial peptides," Mucosal Immunology, vol. 5, no. 5, pp. 501-512, 2012.

[78] M. B. Geuking, K. D. McCoy, and A. J. Macpherson, "The function of secretory $\operatorname{IgA}$ in the context of the intestinal continuum of adaptive immune responses in host-microbial mutualism," Seminars in Immunology, vol. 24, no. 1, pp. 36-42, 2012.

[79] A. J. Macpherson and T. Uhr, "Induction of protective IgA by intestinal dendritic cells carrying commensal bacteria. Science," Science, vol. 303, no. 5664, pp. 1662-1665, 2004. 


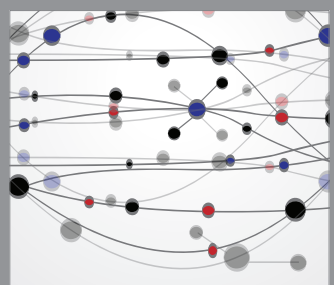

The Scientific World Journal
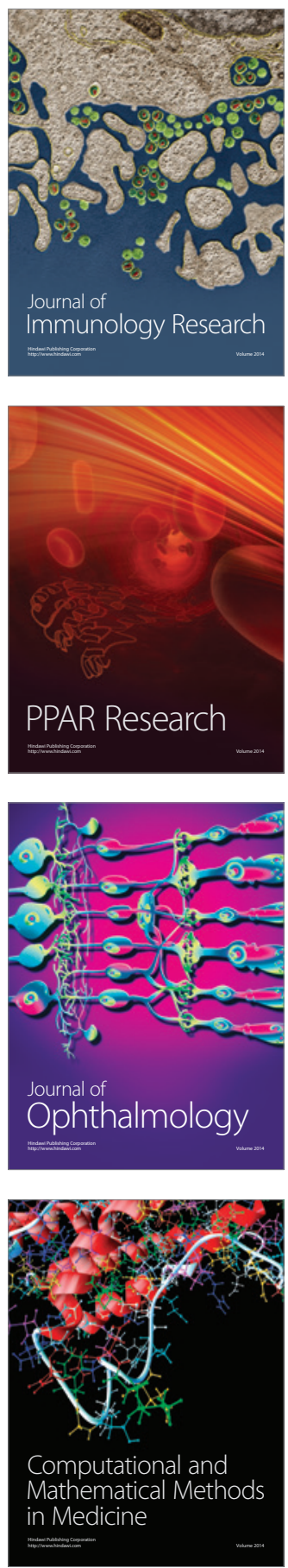

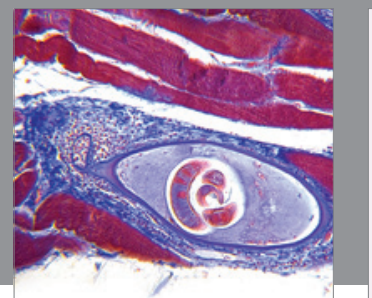

Gastroenterology

Research and Practice
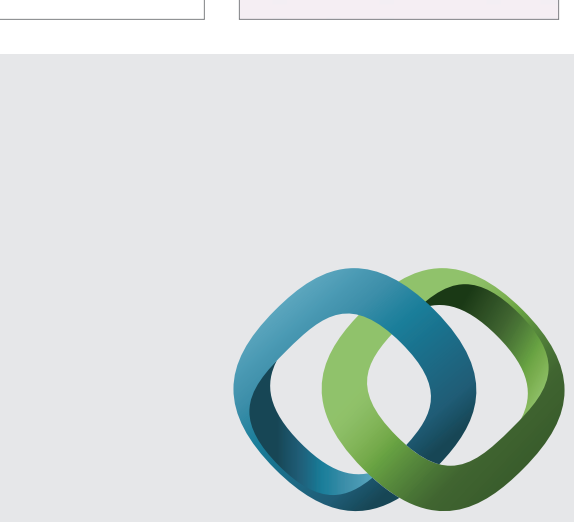

\section{Hindawi}

Submit your manuscripts at

http://www.hindawi.com
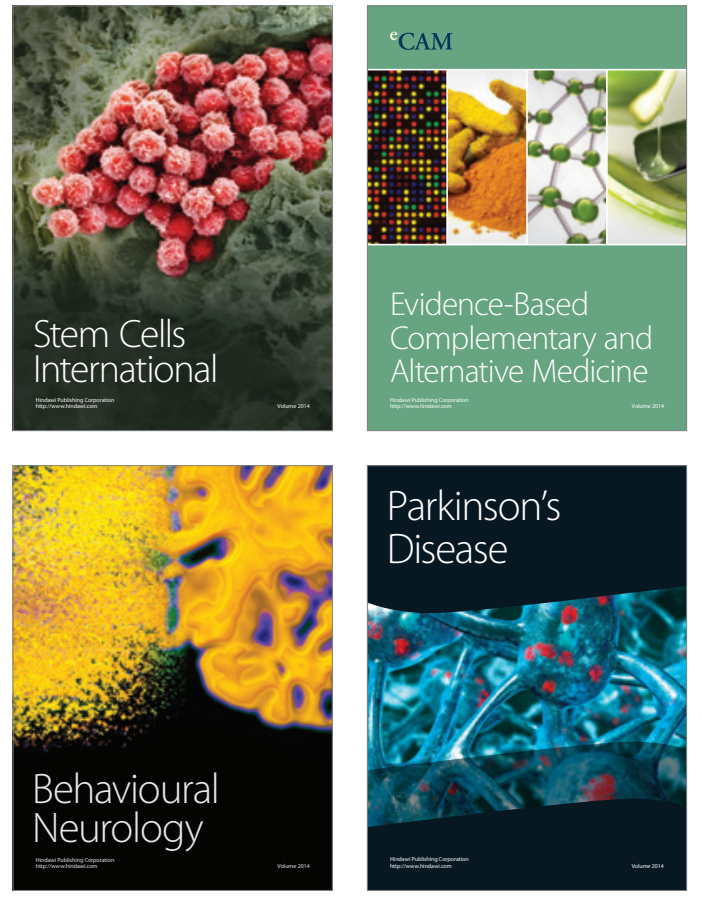
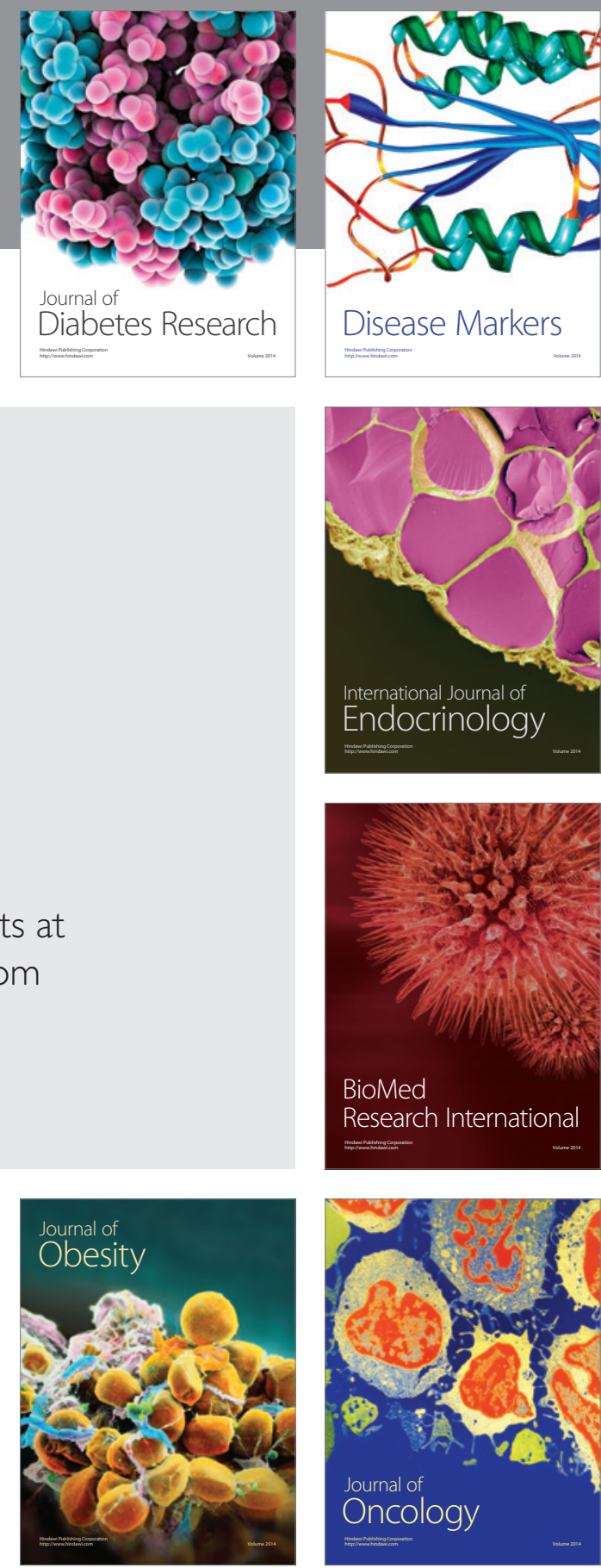

Disease Markers
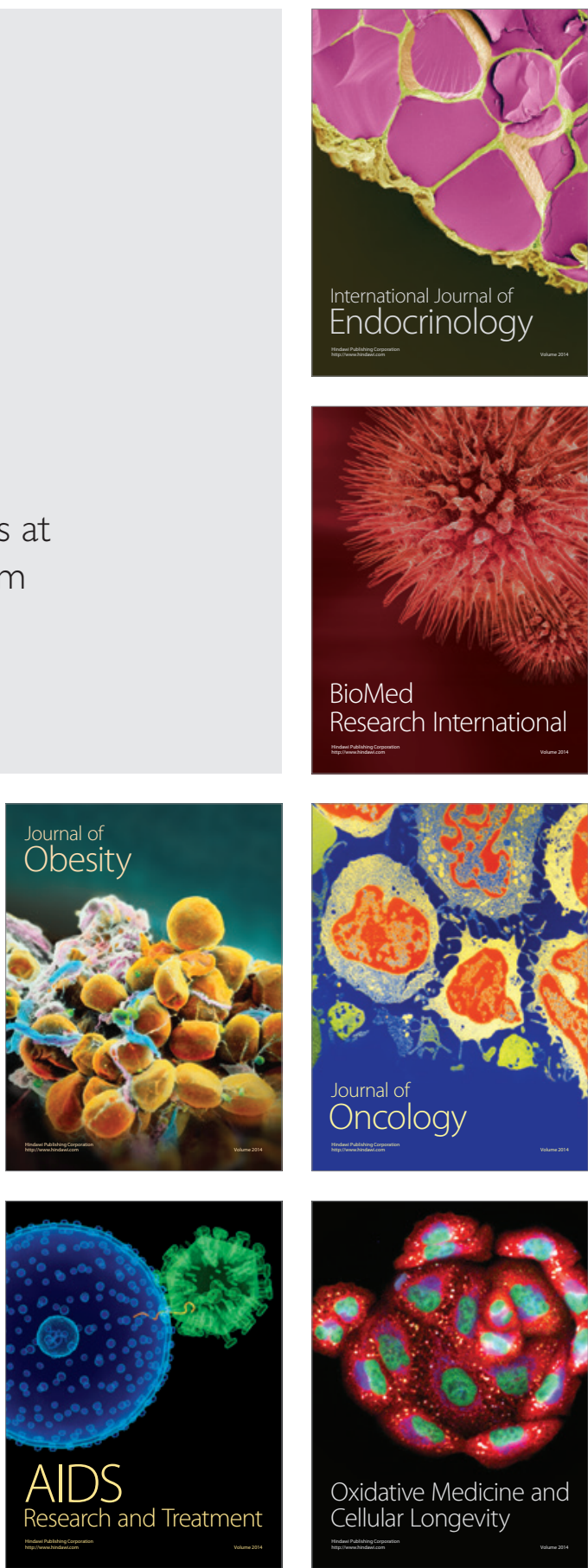